\title{
A Uniaxial Loading Device for Studying Mechanoresponses of Single Plant Cell*
}

\author{
Liqing Zhu', ${ }^{1,2}$, Xue Fu ${ }^{3,4}$, Jie Yan ${ }^{5}$, Junyu Liu ${ }^{5}$ \\ ${ }^{1}$ School of Chemistry and Chemical Engineering, Chongqing University of Science and Technology, Chongqing, China; \\ ${ }^{2}$ College of Bioengineering Chongqing University, Chongqing, China \\ ${ }^{3}$ School of Chemistry and Chemical Engineering, Southwest Petroleum University, Chengdu, China \\ ${ }^{4}$ Key Laboratory of Biorheological Science and Technology (Chongqing University), Ministry of Education, Chongqing, China \\ ${ }^{5}$ College of Bioengineering Chongqing University, Chongqing, China \\ Email: cqzhuliqing@126.com, fuxue1981@163.com,yanjie185@sina.com,Biotljy@163.com
}

Received 2013

\begin{abstract}
A system which consists of a loading chamber unit, displacement sensor, data collector and processor, and a feedback control, was established for applying mechanical forces to single plant cells. The method works by compressing an agar cell-suspension block between parallel surfaces through using a force-feedback control circuit coupled to a microchip, delivering the pre-defined. The actual controlled stimulus is achieved whilst measuring the force being imposed on the cell, and its deformation. The Arabidopsis protoplasts were utilized to test the system. It provides an experimental approach to investigate the mechanoresponses of plant cells in vitro conditions.
\end{abstract}

Keywords: Mechanical Loading; Plant Cell; Chamber; Feedback Control

\section{Introduction}

Plants are unable to escape from an unfavorable environment, they must respond to both endogenous and exogenous stimulus starting with a transduction of mechanical perturbation into a biological signal and ending into a global response as modification of growth, which is known as thigmomorphogenesis [1]. Besides, plants use regulated cell expansion to adapt their form to environmental conditions. In contrast with most non-living material systems, the cell structure is a dynamic system that adapts to its local mechanochemical environment, the mechanical behavior of a living plant cell can't be characterized only in terms of mechanical properties [2]. Observation of the patterns of cell expansion and wall orientation is challenging because it occurs on several scales, which range from the cellular to the tissue level, and ultimately to a consideration of the whole plant. In particular, application of external mechanical stimuli can induce biochemical reactions, including the synthesis of new biomolecules and the enhanced interaction among biomolecules that can generate mechanical forces [3].

Despite the experimental sophistication and computational approaches in cell and molecular biology, the mechanisms by which mechanical stimuli could influence

*Supported by the Science and Technology Project of Chongqing Municipal Education Commission, China (Project No. KJ121403). cell signaling processes are poorly understood [4,5]. In the past few decades, engineering fundamentals have been used to design devices for probing the mechanical properties of living cells [6,7]. Here our focus is on the development of mechanical stimulating system, which provided organismal survival conditions and measurable forces delivery. In the present work, the authors report the use of compressive force generated by a controllable loading system as a tool to investigate cell in situ with the actual stress and alteration of living cells' geometry [8]. Our design of this system will allow us to conduct real-time studies for cellular responses to compress forces on samples. Specifically, we want to satisfy the following criteria:

1) The system has to be small enough to be allowed conventional and fluorescence microscopic observation at high resolution.

2) The system should be a sustainable plant cell culture platform which can support the regeneration of a small portion of cells in a long term culture.

3) The precision of linearity of displacement sensor for the system has to be accurate enough to be achieved to conduct accurate compress stress analysis with an upper limit of $300 \mathrm{mN}$.

\section{Equipment and Method Development}

1) System Design

The basic loading technique was that described by 
Lintilhac et al. [9]. An in-vitro experimental systems are capable of generating controlled and well-defined mechanical forces were developed, which as a typical of micro loading and measuring system, the basic components of this apparatus are: loading unit, displacement or deformation sensor, data collector and processor, and feedback control. The conceptual simple schematic of structure of the system is shown in Figure 1.

2) Device Fabrication

The chamber of the apparatus was constructed consisting of one $2.0 \mathrm{~mm}$ thick deck of cast acrylic $(30.0 \mathrm{~mm} \times$ $90.0 \mathrm{~mm}$ ).

The deck has one threaded holes, in which a connecting rod was set for connected to the stepper motor. To enable accurate, sustainable application of forces, a stepper motor (57BYG250A, stepper angular distance: $0.9 \%$ $1.8^{\circ}$, operating voltage: $4.5-7.5 \mathrm{~V}$ ) that mounted to the top of pedestal was utilized to automate the movement of applying connecting rod, which served as the source to the deformation of test specimens. The stepper motor has a threaded rotor which engages a threaded lead screw, and the lead screw in turn is attached to a guide rod which could slide freely along the axis of the guide way (to maintain alignment of the slide relative to the medium planar plate). The guide way enable positioning planar motion of the subsequently connected applying rod, applying baffle $(20.0 \mathrm{~mm} \times 15.0 \mathrm{~mm})$ that can be gradually and accurately energized to move toward and away from the vertical slide of the sample in order to provide a direct forces (ranged from 50 to $300 \mathrm{mN}$ ) to the test specimens disposed in the chamber. A schematic diagram of the apparatus is shown in Figure 2.

3) Feedback control technique

In general, for recording the force-displacement data during the application of stress, sensor to simultaneously monitor load and displacement is required, respectively. Automatic strain measurement described in the work was adopted with half-bridge circuit, which is composed of some strain sensitive electrical resistance strain gauges. The strain gauges (sensitivity factor: $2.12 \% \pm 1 \%$ ) themselves were placed on the surface of a piece of leaf spring, whose one end was anchored on the guide rod and another bolted to a certain position of the applying rod, amplifying and transforming the displacement signal to strain gauges. It is easy to find the relationship between the e maximal actuator strain $\varepsilon_{\max }$ and the force $F$, which applied on the free end of leaf spring:

$$
\left|\varepsilon_{\max }\right|=\left|\frac{6 F l}{E b h^{2}}\right|
$$

Where $E$ is its elastic modulus, $l, b$ and $h$ are its length, width and height of the rectangular section of leaf spring, respectively. When the external forces are applied, the maximal strain will be produced close to the root of leaf spring. Equation (1) can be used to obtain the consequential strain agar.

The altered resistance signals were collected and regulated by the strain indicator (BZ2201, Beidai River Land Science and Technology Ltd.), and then transferred to the control and monitoring subsystem, thereby the displacement could be recognized directly from the test specimens into a data acquisition system. Specifically, the single channel dynamic strain indicator is a versatile,

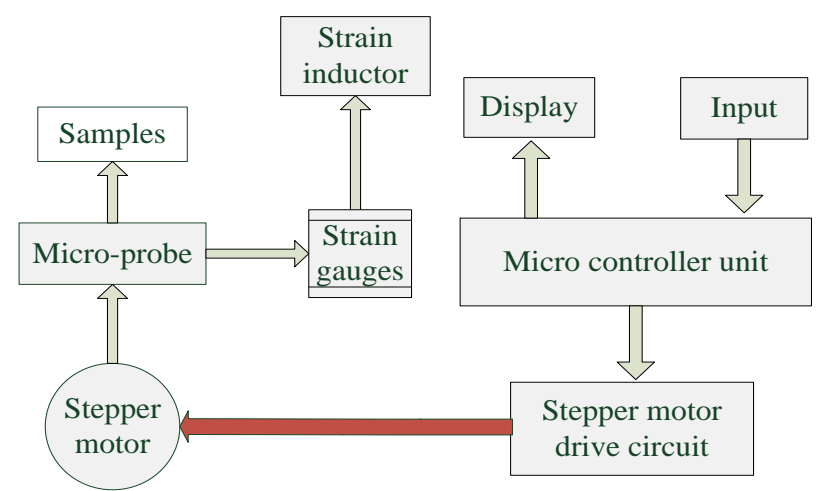

Figure 1. Block diagram of simple structure of the system.

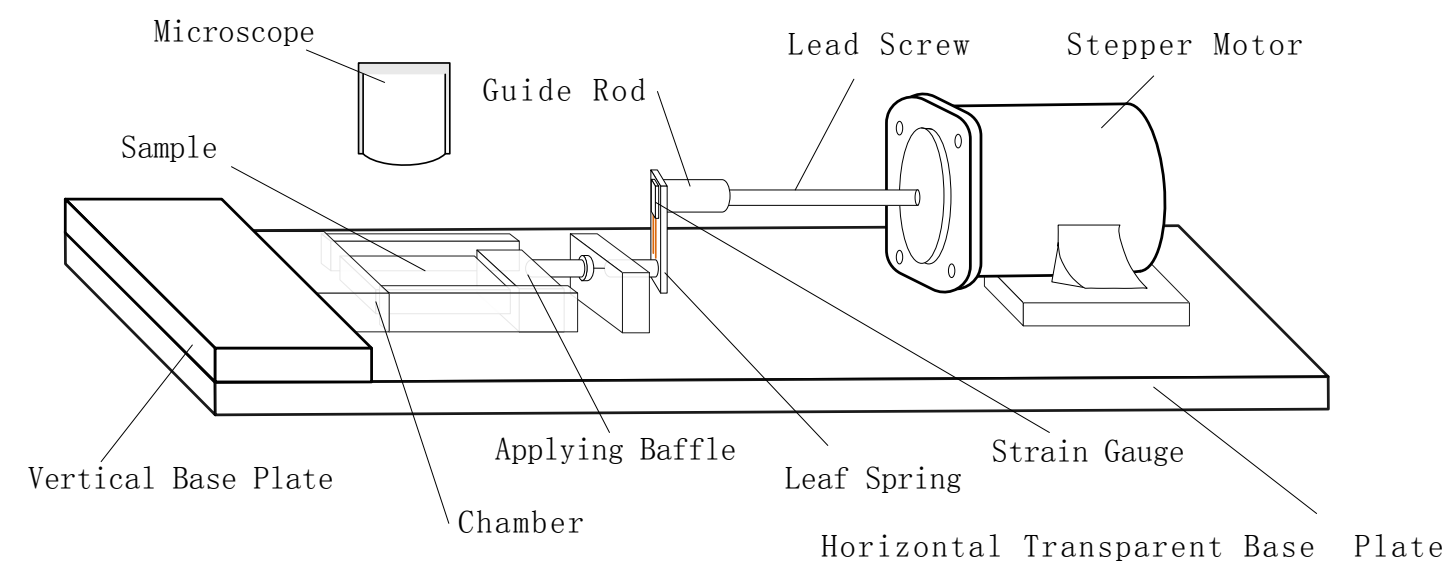

Figure 2. Schematic diagram of the apparatus. 
high-precision laboratory-type instrument developed for use with strains gauges and strain gauge-based transducers to correcting for the spread in gauge resistance and gauge factor. For overcoming the circumstances and to compensate the thermal effect, temperature compensation circuit was designed mainly by means of two external temperature compensating gauges.

The micro-controller unit (MCU) based closed-loop control subsystem was built to measure and manipulate this device. The data acquisition and control unit consists of a multifunction single chip (SPCE061A, Sunplus Technology Company Limited) [10], which provides seven channel 10-bit high-speed analog-to-digital converters (ADC), and two 10-bit digital-to-analog converter (DAC) output channels, combined with higher processing speed (up to $49.152 \mathrm{MHz}$ ). The voltage regulator SPY0029 was utilized as power source to provide $3.3 \mathrm{~V}$ d.c. based on the operating voltage of single chip that ranged from 2.6 to $3.6 \mathrm{~V}$. The input signal comes from a microchip program based on the DAC, it could preflee the desired force level. In addition, the I/O channel provides output electronically control as well as impulse wave signal. A four phase stepper motor component is driven to deliver and maintain a desired precise force with the uniaxial movement. This feedback control circuit provides a realtime visual indication on the digital display of the strain indicator output, the signal of the strain gauge and the control signal would be compared while the force is sensed.

\section{System Test}

The loading system must be calibrated by verifying the accuracy and precision of linearity of the displacement sensor. To verify this device, the protoplasts isolated from the wild-type Arabidopsis thaliana (ecotype Columbia Col-0) rosette leaves were retained as experimental material, Protoplasts were then immobilized by gently swirling them into low-melting-point agar in MS medium supplemented with $0.4 \mathrm{~mol} \cdot \mathrm{l}^{-1}$ mannitol, $0.1 \% \mathrm{MES}$, sucrose, 2,4-dichlorophenoxyacetic acid, and self-conditioned medium harvested from suspension cultured Arabidopsis cells [11]. Then the loaded test specimen which embedded with living cells was removed and chipped into slices of approximately $2.0-5.0 \mathrm{~mm}$ thick, and it was placed onto the chamber of the mechanical loading apparatus described above.

Hamant has reported that microtubule orientation in the shoot apical meristem was found to follow the orientation of stress patterns in the organ [12], this conclusion supports the prior viewpoint that the local microinduction of expansin expression and resulting cell wall softening is sufficient to induce morphogenetic processes, leading to the initiation of leaf structures from the shoot apical meristem [13]. Continuous uniaxial compressive force (at approximately 50 - $300 \mathrm{mN}$ ) was imposed on the opposite sides of agar block, time ranged from 60 seconds to 1200 seconds, this allowed a microscope to be brought close to the cell under test. Our device could play a role in the relative research field. The applying baffle displacement and the cell deformation could be observed, and a force-deformation curve generated. As shown in Figure 3, the deformation of embedded protoplasts under constant mechanical strain is visible in the substrate, the minor axis will be the potential division plane. A $20 \%$ stain of the agar matrix could induce a significant deformation of the single cell without cell wall (Figure 4; control versus loaded). It should be noted that the protoplast all show a preferential orientation (Orientation = angle between stress vector and minor axis) with their long axis perpendicular to the principle stress vector, and a 15-mins loading of such intensity did not influence the viability of the subjected protoplasts in the subsequent 24h's culture.

\section{Results and Discussion}

An instrument, which has potential to provide mechanical property information at the individual cell Level has been devised that is capable of delivering static or cyclic compression or tension of variable duration to cells cultured in vitro. A lack of published data on properties of Arabidopsis protoplasts prevents a comparison of the

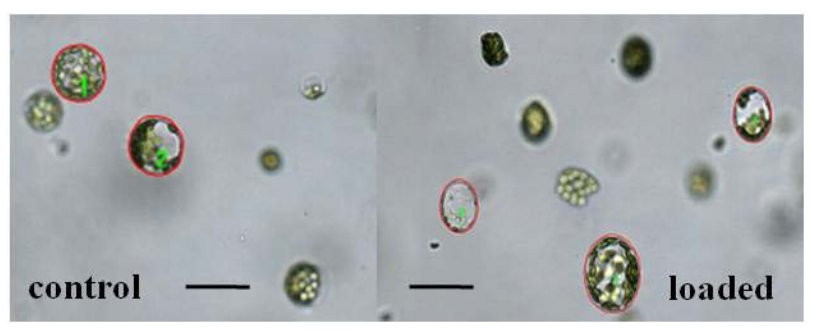

Figure 3. Control experiment to test the influence of the mechanical stimuli to the protoplasts elongation. Contrast control group, the loaded group show that the aspect (Aspect $=$ minor axis/major axis) of embedded protoplasts altered significantly. Bar $=50 \mu \mathrm{m}$.
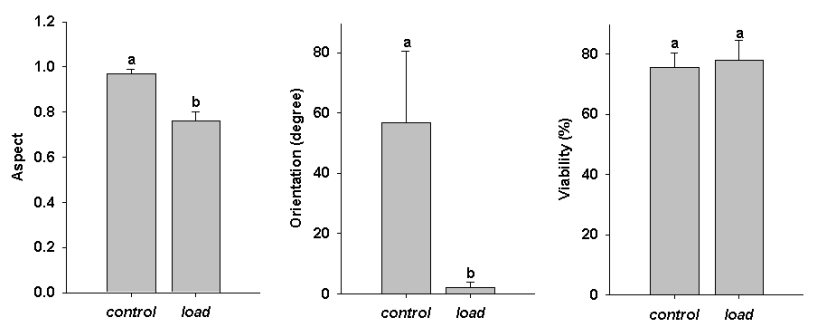

Figure 4. 20 mins constant mechanical strain $(80 \%)$ induced protoplast deformation-Aspects, Orientation and Viability. Compare the orientation of loaded group relative to control group, it is reduced significantly. Otherwise, the viability of both groups rarely changed. 
other parameters. However, the values of this method should be a function of the state of the cell will allow us to model mechanical stress on cell and cytoskeleton in studying plant cell mechanoresponses.

\section{Acknowledgements}

The authors thank Chongqing University of Science and Technology for financial support for this work.

\section{REFERENCES}

[1] M. Jaffe and S. Forbes, "Thigmomorphogenesis: The Effect of Mechanical Perturbation on Plants," Plant Growth Regulation, Vol. 12, No. 3, 1993, pp. 313-324. http://dx.doi.org/10.1007/BF00027213

[2] A. Geitmann and J. K. E. Ortega, "Mechanics and Modeling of Plant Cell Growth,” Trends in Plant Science, Vol. 14, No. 9, 2009, pp. 467-478. http://dx.doi.org/10.1016/j.tplants.2009.07.006

[3] M. A. Wozniak and C. S. Chen, "Mechanotransduction in Development: A Growing Role for Contractility,” Nature Reviews Molecular Cell Biology, Vol. 10, No. 1, 2009, pp. 34-43. http://dx.doi.org/10.1038/nrm2592

[4] J. Rajagopalan and M. T. A. Saif, "MEMS Sensors and Microsystems for Cell Mechanobiology," Journal of Micromechanics and Microengineering, Vol. 21, No. 5, 2011. http://dx.doi.org/10.1088/0960-1317/21/5/054002

[5] V. Chickarmane, A. H. K. Roeder, P. T. Tarr, A. Cunha, C. Tobin and E. M. Meyerowitz, "Computational Morphodynamics: A Modeling Framework to Understand Plant Growth,” Annual Review of Plant Biology, Vol. 61, 2010, pp. 65-87. http://dx.doi.org/10.1146/annurev-arplant-042809-112213

[6] K. J. Van Vliet, G. Bao and S. Suresh, "The Biomechanics Toolbox: Experimental Approaches for Living Cells and Biomolecules," Acta Materialia, Vol. 51, No. 19, 2003, pp. 5881-5905.

http://dx.doi.org/10.1016/j.actamat.2003.09.001

[7] T. M. Lynch and P. M. Lintilhac, "Mechanical Signals in Plant Development: A New Method for Single Cell Studies,” Developmental Biology, Vol. 181, No. 2, 1997, pp. 246-256. http://dx.doi.org/10.1006/dbio.1996.8462

[8] J. Zhou, B. C. Wang, Y. Li, Y. C. Wang and L. Q. Zhu, "Responses of Chrysanthemum Cells to Mechanical Stimulation Require Intact Microtubules and Plasma Membrane"Ccell Wall Adhesion,” Journal of Plant Growth Regulation, Vol. 26, No. 1, 2007, pp. 55-68. http://dx.doi.org/10.1007/s00344-006-0029-2

[9] P. M. Lintilhac and T. B. Vesecky, "Stress-Induced Alignment of Division Plane in Plant Tissues Grown in Vitro," Nature, Vol. 307, 1984, pp. 363-364. http://dx.doi.org/10.1038/307363a0

[10] Y. F. Luo, "Sunplus 16 Bit Microcontroller Application Foundation,” Beihang University Press, 2003.

[11] S. D. Yoo, Y. H. Cho and J. Sheen, “Arabidopsis Mesophyll Protoplasts: A Versatile Cell System for Transient Gene Expression Analysis,” Nature Protocols, Vol. 2, No. 7, 2007, pp. 1565-1572. http://dx.doi.org/10.1038/nprot.2007.199

[12] O. Hamant, M. G. Heisler, H. Jönsson, P. Krupinski, M. Uyttewaal, et al., "Developmental Patterning by Mechanical Signals in Arabidopsis,” Science, Vol. 322, No. 5908, 2008, p. 1650. http://dx.doi.org/10.1126/science.1165594

[13] S. Pien, J. Wyrzykowska, S. McQueen-Mason, C. Smart and A. Fleming, "Local Expression of Expansin Induces the Entire Process of Leaf Development and Modifies Leaf Shape," Proceedings of the National Academy of Sciences, Vol. 98, No. 20, 2001, p. 11812. http://dx.doi.org/10.1073/pnas.191380498 